\title{
Talento, experto o las dos cosas
}

\author{
Franchek Drobnic ${ }^{\text {y Julio Figueroa }}{ }^{\mathrm{b}}$
}

aDepartamento de Fisiología del Deporte del Centro de Alto Rendimiento. Sant Cugat del Vallés. Barcelona. España. Servicios Médicos del FC Barcelona. Barcelona. España.

'Departamento de Ciencias de la Actividad Física y Deporte. Facultad de l’Educació. Universitat de Vic. Vic. España.

\section{RESUMEN}

En ocasiones es difícil discernir entre el concepto de individuo con talento e individuo experto. Sin duda se debe a que los roles que ofrecen los individuos que alcanzan el éxito en el deporte, la música, el teatro, la literatura, etc., son una muestra de ambas condiciones: una si se quiere innata, el talento, y otra adquirida, la de ser experto. También, a veces, desearíamos que los talentos trabajasen para además ser expertos, aspecto que los elevaría a un grado superior de excelencia.

En el presente artículo se realiza una puesta al día de ambos conceptos con el objeto de establecer una base adecuada sobre la que se sostengan con mayor solidez las decisiones y actuaciones del formador. También se presentan algunas nociones sobre el seguimiento y control de los muchachos que disfrutan en las canteras deportivas siempre con la orientación básica de formar individuos completos con un rendimiento óptimo.

PALABRAS CLAVE: Talento. Experto. Entrenamiento. Cantera.

\section{ABSTRACT}

Distinguishing between the concepts of talent and expertise is sometimes difficult. This is undoubtedly because persons successful in sports, music, acting and literature, etc. show a mixture of both qualities, talent being innate while expertise is acquired. Sometimes we would also wish that talented individuals would apply themselves to becoming experts, which would allow them to achieve a higher degree of excellence.

The present article provides an update on both concepts with a view to establishing a solid base for trainers' decisions and interventions. Some notions on following the progress of boys participating in sports academies are presented from the perspective of producing well-rounded individuals with optimal performance.

KEY WORDS: Talent. Expertise. Training. Sports academy.
Se entiende como talento la facultad o grupo de facultades con una cierta especificación, superior a la media, pero que debe aún manifestarse. El talento específico en el deporte es el que hallamos en el individuo que tiene unas cualidades motrices superiores a las normales para realizar un tipo de actividad determinada y que dispone a su vez de una superioridad intelectual y de actitud positiva. Aunque es cierto que estas dos últimas características -inteligencia y actitud- son menos visibles, no son despreciables y deben valorarse y potenciarse, pues son determinantes en el rendimiento final del deportista.

Uno de los aspectos importantes en el buen funcionamiento de un club deportivo es poder disponer de secciones de deporte base que trabajen en todos los ámbitos que competen al desarrollo del talento deportivo, su descubrimiento, valoración, apoyo y seguimiento.

El término "talento" se refiere al mensaje de la parábola de los talentos (Mt 25:14-30), donde se expone que siempre debemos demostrar la disposición en potenciar aquellas cualidades que nos son concedidas, pretendiendo que se expresen y produzcan en su máximo provecho. La definición del término según el Diccionario de la Real Academia Española (2001) indica que es una palabra que proviene del latín talentum, y éste del griego $\tau \lambda \alpha \nu \tau o \nu$, que significa plato de la balanza o peso, pues era una moneda de cuenta de los griegos y de los romanos. Su acepción en el idioma español indica tres definiciones: capacidad de entender, capacidad para el desempeño o ejercicio de una ocupa-

El presente artículo es una actualización del capítulo del libro "Bases científicas para la salud y el óptimo rendimiento en el baloncesto" de la editorial Ergon, redactado por los mismos autores.

Correspondencia: Franchek Drobnic. Departamento de Fisiología del Deporte. Avda. Alcalde Barnils 3-5. 08I73 Sant Cugat del Vallés. Barcelona. España. 
ción y, por último, es el concepto de una persona inteligente o apta para determinada ocupación. En definitiva, es "el conjunto de dones naturales y sobrenaturales con que Dios enriquece a los hombres" (diccionario de la Real Academia, 1984).

Existen dos hipótesis generales sobre el concepto. Por un lado, la de que es un individuo con una carga genética que le ofrece unas posibilidades superiores frente a sus congéneres y que pueden ser de orden físico -como la velocidad, la fuerza o la habilidad, entre otras- o bien de orden psíquico -capacidad competitiva, gestión de emociones, capacidad de reacción y la búsqueda de recursos, etc. La otra tendencia es la de los sujetos que aprenden una serie de cualidades a lo largo de su desarrollo basadas en la experiencia y el correcto aprendizaje y que les son positivas y forman de una manera óptima. Es evidente que el concepto actual, si se quiere "popular", de talento se basa en ambas ideas. El talento está dotado de atributos o características especiales que le hacen desarrollar, aprender y ejecutar habilidades con mayor facilidad que a los otros individuos de su entorno en las mismas condiciones. Sin embargo, el talento por sí solo no puede alcanzar el éxito, o al menos de una forma continuada. Como indica Platonov-citado por diversos autores-, "el éxito sólo puede ser fruto del desarrollo del talento a través de un trabajo duro y juicioso en un contexto social favorable". Más adelante se explicarán estos aspectos.

Una vez definido el concepto de talento y su disposición, es referencia obligada los tipos de talento según el modelo de $\mathrm{Hahn}^{1}$, para quien existen tres tipos:

- Talento motor general. Supone una gran capacidad de aprendizaje motor que conduce a un dominio motor más fácil, más seguro y más rápido, y a un mayor repertorio motor aplicable de modo más diferenciado.

- Talento deportivo. Disposición por encima del promedio de someterse a un programa de entrenamiento deportivo para conseguir éxitos deportivos.

- Talento específico para un deporte. Indica qué requisitos físicos y psíquicos son necesarios para poder alcanzar rendimientos extraordinarios.

Pero identificar los componentes que configuran un talento deportivo no es fácil. Existen aproximaciones en este y en otros deportes, individuales y de equipo, para reconocer ciertas aptitudes que puedan identificar al futuro profesional. Las aptitudes para una actividad compleja varían durante el desarrollo físico del individuo, y éstas aumentarán o no en función del aprendizaje impartido en cada período. En la tabla I se mues-
Tabla I Áreas que contribuyen al rendimiento deportivo

Condición física general, estado de salud

Características fisiológicas del individuo

Características psicológicas del individuo

Capacidad de tomar decisiones técnicas

Habilidad personal en el esquema de juego

Características sociológicas del individuo

tran algunos ámbitos donde hallar las características que determinan un buen rendimiento deportivo.

Cuando un técnico-observador determina que este o aquel muchacho es un futuro gran jugador, lo refiere a una serie de cualidades -físicas, técnicas, tácticas, sociales y humanas- relacionadas con el deporte que se perciben del deportista y se adecuan a unas referencias estructuradas en el bagaje de conocimientos técnicos y experiencia del profesional que les asiste.

Existe un factor importante en el jugador de deportes de equipo, que se refiere a la capacidad para el juego complejo ${ }^{2}$. Esta cualidad comprende los cuatro elementos básicos: la condición física, las capacidades técnica y táctica, y las habilidades psicológicas. Esta capacidad no es mensurable con cronómetros ni cintas métricas, tampoco a través de tantos marcados o evitados, ni con pruebas individuales o una serie de baterías conocidas hasta la fecha. Según este autor, los aspectos distintivos del jugador de equipo con capacidad para el juego complejo se contemplan en las siguientes capacidades:

- Disposición para el rendimiento.

- Aceptación del riesgo y los retos en condiciones adversas.

- Perseverancia y continuidad en las acciones.

- Reacciones adecuadas a las situaciones cambiantes.

- Facilidad y fluidez de movimientos.

- Capacidad de anticipación a las situaciones del juego.

- Conocimiento profundo de sus puntos fuertes y débiles.

Todas son características subjetivas desde un punto de vista tanto cuantitativo como cualitativo, pero pueden definirse con el fin de confeccionar un modelo que oriente al futuro comité de selección del club en la necesidad de adquirir o no determinado jugador, y pueden servir de modelo de seguimiento de la evolución del sujeto; si no a nivel general del deporte, sí por lo menos para un orden interno. 
En ese sentido, es lógico que cada club tenga unas prioridades, por su esquema de juego, la tradición deportiva, o por simples intereses o necesidades derivadas de deficiencias en puestos determinados. Los hay que desean jugadores altos, otros rápidos, otros los quieren fuertes, otros muy técnicos, con buen dominio de la pelota, que sepan jugar en equipo, con disposición para aprender, ambición, etc. En general, se considera que deben primar las cualidades técnico-tácticas sobre las físicas o fisiológicas, aunque es defendible cualquier opinión si se dispone de un objetivo claro y sincero y se propone el método para apoyar y reconducir las deficiencias evaluadas en los diversos ámbitos que conformarán el buen jugador.

En cualquier caso, cuando se valora un talento se pretende hallar aquel sujeto con cualidades técnico-tácticas, con una cierta proyección física y con unas capacidades volitivas importantes.

\section{TALENTO O EXPERTO}

Los investigadores que se dedican a identificar los factores que distinguen lo excepcional de lo ordinario han evaluado diversas teorías para el desarrollo de un experto. Éste es un término, el de "experto", que viene utilizándose cada día más. Conceptúa a aquel individuo que no sólo dispone de un don especial -ya se descubrirá en su día si genético o no-, sino que tiene que estimularlo para alcanzar el máximo rendimiento.

Los individuos catalogados de expertos que atesoran una amplia experiencia, adquieren un elevado número de patrones complejos que después utilizan para almacenar nuevos conocimientos sobre qué acciones deberán realizarse en situaciones similares. Este aspecto le confiere otra acepción: un deportista puede no recibir cierto número de "talentos" o "estar dotado" como sus compañeros. Es cierto que los factores genéticos intervienen en mayor o menor medida, pero lo que está claro es que los de origen ambiental, o fenotípicos, desempeñan un papel importante en la variación interindividual frente al deporte $\mathrm{y}$ al deporte en equipo.

Se requieren altos niveles de entrenamiento para alcanzar el grado de experto. Se calcula que un período cercano a los 10 años de práctica de calidad, basándose en la regla de los 10 años de Simon y Chase ${ }^{3}$ y el poder de la práctica según Newell y Rosenbloom ${ }^{4}$. Los jugadores expertos son aquellos que organizan la información de una forma más precisa y eficaz. No disponen de una memoria superior, pero la gestionan mejor porque utilizan recursos aprendidos con eficacia. En ese sentido, el jugador experto tiene unas características muy bien determinadas (tabla II).

\section{Tabla II Características que distinguen a los expertos}

Mayor conocimiento de tareas específicas

Mayor capacidad para recibir y percibir información

Mayor capacidad de gestionar esa información

Capacidad en reconocer fácilmente patrones de juego

Utilizar mucho mejor las probabilidades de las jugadas

Tomar decisiones más rápidas y más apropiadas

La regla de los 10 años nos informa que se precisan 10 años de alto nivel de entrenamiento para alcanzar la excelencia en cualquier materia. Se ha demostrado en diversos ámbitos: música, matemáticas, natación, fondo en carretera, tenis, etc. No es sólo entreno, es decir, horas de trabajo, sino invertir en un entreno específico, deliberado, personal, encaminado a la mejora en el deporte. Ésta es la parte que se debe al "poder de la práctica”. Pero ¡atención! No es sólo el tiempo, sino calidad. El nivel de rendimiento final se determina por la cantidad de tiempo invertido en una "bien definida tarea con una dificultad apropiada para el nivel particular individual, en la que se reciba un feedback de información y oportunidades para la repetición y corrección de errores"s. Por eso, la calidad del entrenamiento debe estar siempre por encima del criterio de acumular horas, sobre todo una vez alcanzado un nivel deportivo; en ese momento la habilidad del entrenador para diseñar el entrenamiento es un factor crítico ${ }^{6}$. Varios aspectos que no es habitual observarlos en los entrenamientos de ciertos deportes de equipo, como sí lo es en los deportes de precisión e individuales. Se sabe que los deportistas expertos o así considerados por ser internacionales, dedican más tiempo a su actividad deportiva y a actividades específicas y relacionadas con el rendimiento deportivo (sesiones técnicas, tácticas, visualizaciones, correcciones...) que los que no lo son ${ }^{7}$.

\section{Selección del talento deportivo}

Para poder alcanzar el nivel de jugador excelente, en base a la selección de talentos deportivos, también se debe poseer una estructura de club y de lo que se conoce como "cantera". Este término pretende identificar un talante y una forma de trabajar, además de un espacio donde hacerlo bien. Una estructura basada en una disciplina, que identifica con claridad una serie de objetivos y resultados para cada grupo de edad. Que permi- 
tirá realizar un buen seguimiento de los talentos adquiridos, evaluar sus mejoras y deficiencias, corregirlas a medida que cambia de categoría, en la pretensión de alcanzar la excelencia final. Esta afirmación implica que el equipo técnico defina y determine qué es para él un talento para poder ponerse en marcha en su detección o identificación y sobre todo en su potenciación.

En ocasiones disponemos de individuos que son excelentes en su división, y por diversos motivos se requieren e introducen en categorías superiores. Este hecho, que inicialmente agrada a todos -seguidores del equipo, periodistas, técnicos y al protagonista-, no siempre es lo beneficioso que deseamos. En qué medida puede afectar el futuro rendimiento del jugador que llega a un primer equipo, o cuáles serán las deficiencias que hayamos provocado por ir demasiado rápido, es una verdadera incógnita. La altura y la fuerza demostrada en las acciones, la velocidad en el juego, en la toma de decisiones, los compañeros de diferente madurez, condicionarán las acciones del deportista. En categorías inferiores, donde la madurez física y técnica muchas veces adelanta a la mental, los fracasos no son inhabituales, y no es fácil en algunos de ellos recuperar la confianza del individuo en sí mismo y en el equipo técnico. Hasta este hecho debe ser siempre considerado. Entre otras cosas, y a veces lo olvidamos, por el bien del jugador como ser humano que es, y por el del equipo y el club que invierte un tiempo en hacer valer sus "talentos".

La mejora de nivel debe ser paulatina, permitiendo una adaptación que encauce las virtudes técnicas del muchacho en el nuevo grupo y en las nuevas cualidades del jugador de cada categoría. La diversión, el juego, el contacto social es básico en esas primeros lances. Después, el trabajo físico, técnico, táctico hasta alcanzar el alto rendimiento deportivo. Recordemos que la mejora de las aptitudes depende del aprendizaje, y éste es multifactorial, precisa etapas. El talentoso las puede recorrer más rápido, pero no debería obviarlas si quiere alcanzar el nivel de experto en una materia. En la figura 1 se muestra una clasificación simple de cómo se puede periodizar el entrenamiento en función de la edad del individuo y de ciertas características solicitadas por el juego ${ }^{8}$. En este sentido, la estructura y los contenidos de los entrenamientos y juegos que realiza el deportista van evolucionando a lo largo de su desarrollo. Las actividades consideradas más adecuadas en los últimos estadios de desarrollo del jugador de equipo no tienen nada que ver en cuanto a las actividades, e incluso en cuanto al entorno motivacional, que deben realizar los niños en sus primeros estadios de iniciación deportiva. Se plantea una evolución en dichas actividades, diferenciando cuatro modelos de actividad':

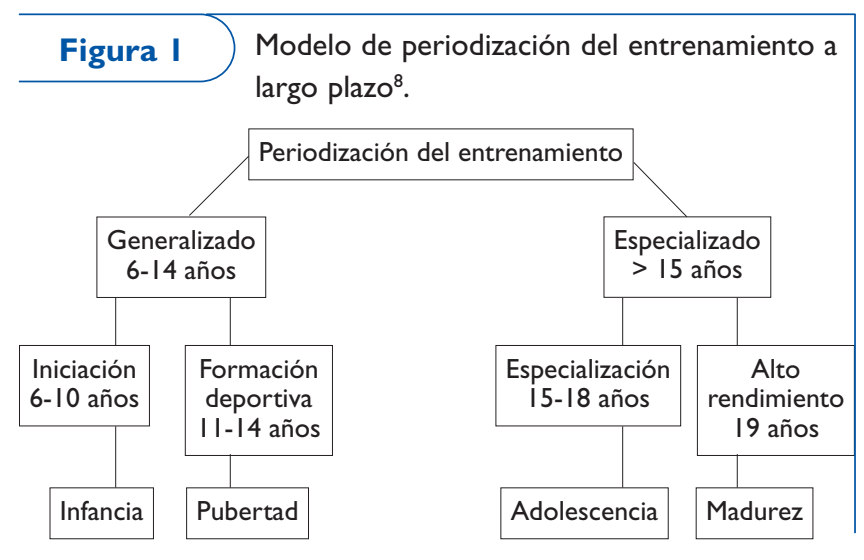

- Juego libre. Se caracteriza por la diversión, por no estar controlado por ningún monitor ni entrenador, no existir correcciones, y porque el niño se centra fundamentalmente en el proceso, obteniendo un placer inmediato y siendo inherente el carácter divertido del juego.

- Juego deliberado. De características similares al anterior, pero en el que ya existe un monitor que aporta algunas orientaciones. Este tipo de actividad caracteriza fundamentalmente a los deportistas durante los primeros años, hasta aproximadamente los 12 años.

- Entrenamiento estructurado. Se caracteriza ya por una orientación hacia la mejora del rendimiento y, por tanto, se centra en el resultado. Está regulado por un entrenador que ofrece correcciones.

- Entrenamiento deliberado. Similar al anterior, pero con una planificación más cuidadosa del entrenamiento. En este caso, la gratificación que se obtiene por implicarse en dicha práctica no es inmediata y es fundamentalmente de carácter extrínseco. Se observa a partir de los 16 años.

La mayor parte de las veces se subestima la edad de aparición del talento deportivo en la alta competición. Es frecuente aducir que los grandes jugadores debutaron como profesionales a edades tempranas, cuando en general deban cubrirse unas expectativas de aprendizaje que sin duda no pueden esperarse hasta los 22 o 24 años. No en vano sería la edad que correspondería a un experto según las propuestas de 10 años de trabajo bien programado y de la que se habló anteriormente. Por otra parte, se considera que la excelencia como jugador no ocurre antes de los 22-25 años, edad en la que se considera a los jugadores maduros, en términos deportivos. La edad de debut o ficha por el primer equipo no debe ser un objetivo, ni tiene que ser un valor añadido. Depende de factores que pueden no 
tener mucho que ver con el jugador, como una política de fichajes determinada, un vacío en cierta demarcación, etc. Valorar ese aspecto, sobre todo por parte del deportista y su entorno, puede ser un grave error en el futuro deportivo del aspirante a experto. Son muchos los jugadores que debutaron, debutan y debutarán en el profesionalismo a edades muy tempranas, con una gran calidad, y por diversos motivos no alcanzaron ni alcanzarán el nivel esperado. Quizá uno de los motivos pudiera ser una excesiva prisa en aprovechar el talento, $y$ una falta de horas de trabajo de calidad.

\section{CONDICIÓN FísıCA}

Es habitual leer en los documentos de educación física y de deporte las pruebas físicas básicas para evaluar al deportista. Debe entenderse el objetivo de las mismas, tal como se expresa: evaluar el estado de forma general y de determinadas manifestaciones fisiológicas ante el ejercicio. Las diversas pruebas pretenden ubicar al jugador en unas características propias de su momento de la temporada, de su edad, de su desarrollo, etc. Son muy útiles para conocer las deficiencias con respecto a lo que se espera de su deporte, de su categoría o de su posición en el campo. Pensemos que a la hora de desarrollar tests para buscar talentos o controlarlos, debe utilizarse, además de evaluaciones de variables de la condición física general, de otros que midan la pericia en los mismos relacionada con la actividad deportiva. Recordemos que lo físico es entrenable, y también nos interesa ver cómo los talentos evolucionan. Un buen físico permitirá entrenos de mejor calidad, mejor recuperación de los mismos, menos lesiones y, en su caso, facilitar la recuperación de las mismas.

Es cierto que algunos jugadores pueden estar favorecidos desde el punto de vista genético en lo que se refiere a la condición física; sin embargo, está ampliamente contrastado que la respuesta fisiológica al ejercicio es dependiente del entrenamiento regular y de su nivel. En ese sentido, en deportes como el fútbol, el baloncesto, el balonmano, etc., de fuerza, de velocidad y con amplias pausas de recuperación, aspectos como la potencia aeróbica máxima, la capacidad aeróbica o el umbral anaeróbico no son características que puedan determinar la selección de uno u otro individuo. La heterogeneidad antropométrica respecto a los porcentajes corporales (músculo/grasa), dentro de unos niveles de talla y envergadura medios para cada posición, tampoco nos indicarán preferencias en individuos en desarrollo que después, ya maduros, podremos forzar en trabajos específicos. Tampoco podemos esperar que un jugador infantil tenga unos índices de potencia anaeróbica elevados, o

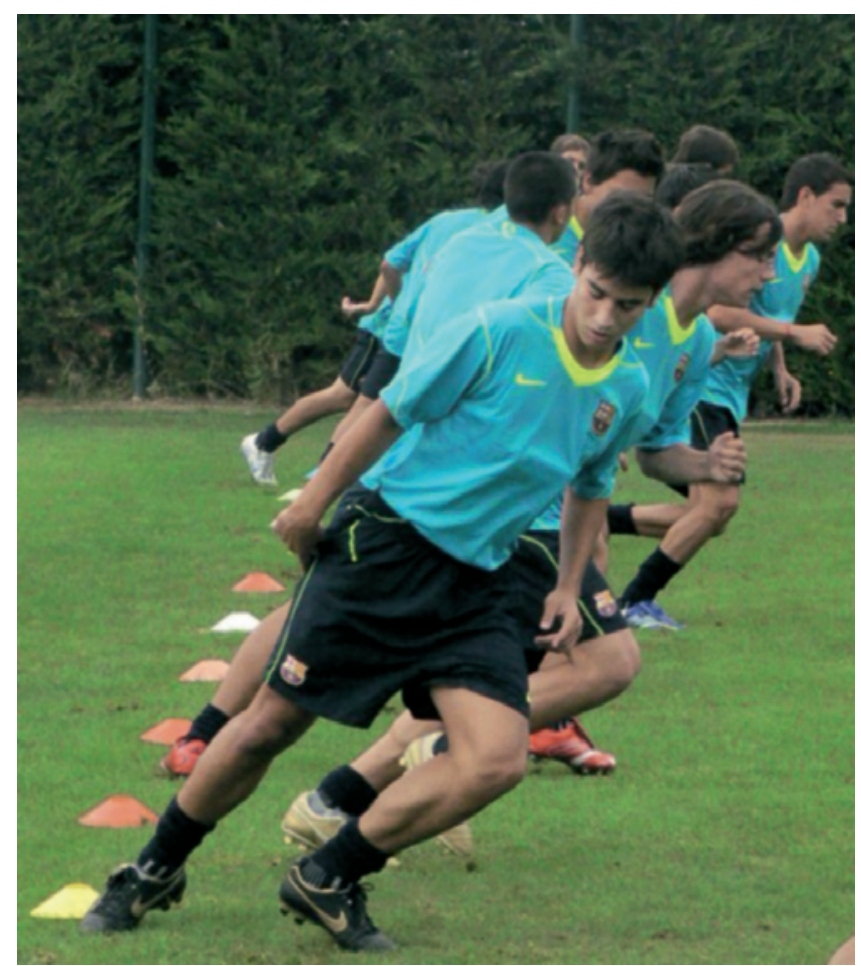

una gran potencia de salto, o una capacidad para realizar sprints de forma repetida, etc., pues su potencia y su capacidad anaeróbica están poco desarrolladas. En cambio, sí será importante que su adaptación al esfuerzo o recuperación cardiorrespiratoria sean correctas, que no tenga alteraciones morfofuncionales que puedan repercutir en su rendimiento futuro, que no padezca una patología de base que no sea recuperable o sea nociva para su salud cuando el rendimiento sea elevado, o que su predicción de talla no se halle dentro de unos rangos de normalidad para su puesto.

En cada edad deportiva las necesidades físicas se modifican y las referencias son más precisas en función de los deseos de excelencia física del modelo exigido en el club. En ese sentido existen baremos como modelo (fig. 2). No los comentaremos aquí, y un buen consejo es que cada club confeccione los suyos en base a sus objetivos y sus posibilidades de control y seguimiento, sin olvidar que las cualidades físicas son entrenables en todas sus manifestaciones. Por eso, las deficiencias descubiertas en cada jugador son siempre mejorables con un trabajo específico y programado. El fracaso de un talento por las cualidades físicas no lo es sólo del deportista, sino también del equipo de seguimiento, de su preparador físico y de su entrenador.

Es una opinión contrastada que los clubes que priman las cualidades físicas se equivocan. Éstas son las menos importantes en determinados momentos del desarrollo. El jugador con 


\section{REVISIONES}

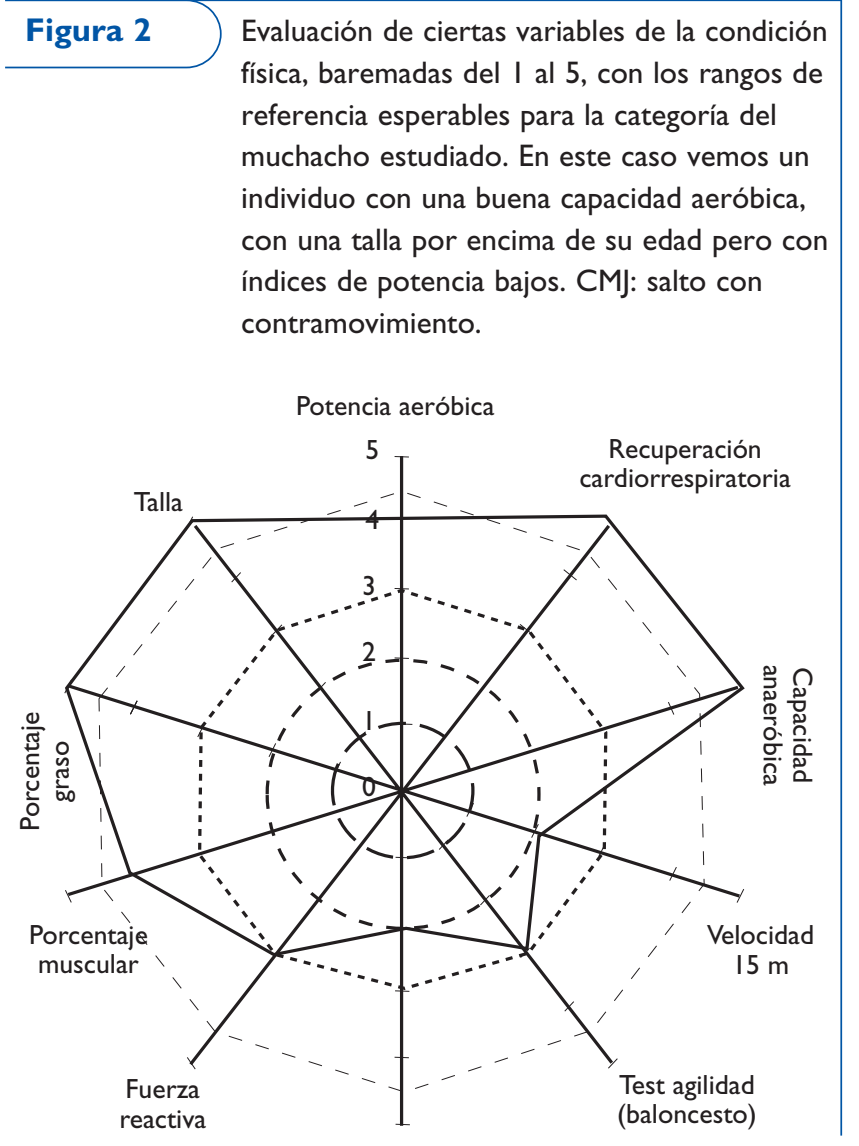

Potencia CMJ

talento al que no se le permite jugar y desarrollar sus habilidades en el terreno competitivo, porque prevalece el objetivo de ganar el partido con jugadores más fuertes o más veloces, nunca evolucionará y se perderá en el banquillo. En ese sentido, debe entenderse que, además de las técnicas, existen cualidades que se entroncan dentro de la esfera psicológica y social del deportista, y que sólo pueden desarrollarse en la competición y no en los entrenamientos. Determinación, agresividad, búsqueda de recursos, cohesión con los demás, etc., son algunos de los muchos aspectos que un jugador demuestra en mayor o menor medida y que aparecerán, crecerán o disminuirán con la guía y el saber hacer del entrenador, de todo el equipo técnico y de los compañeros, pero sólo si se trabaja en ellos.

No debe desprenderse de lo comentado que el vivir un ambiente donde el objetivo inherente a la escuela-club sea el querer vencer en cada lance sea malo o negativo. Ése es el objetivo del juego. Es la dinámica y disciplina de la escuela lo importante. Trabajar en la cantera para formar ganadores del futuro, es decir a largo plazo, o actuar con un objetivo positivo a muy corto plazo. En cada categoría y cada fin de semana.
La opción no es tan bien definida en ningún caso. Los términos intermedios existen, pero no es menos cierto que los ejemplos en uno y otro modelo son claros. Éste es un debate que afecta no sólo a los profesionales que se dedican a la organización, a la gestión, a la investigación, a la enseñanza y al entrenamiento de la mayoría de los deportes, sino también a muchos padres que ven en la actividad deportiva de sus hijos una plataforma de promoción social y económica. La realidad es diferente. En la tabla III se muestran, según Horst Wein ${ }^{10}$, algunos planteamientos de una escuela deportiva, según el deseo de ganar o formar jugadores y aplicable a cualquier deporte.

\section{CONDICIONES GENÉTICAS}

Este aspecto se relaciona sobre todo con las cualidades físicas generales. En lo que respecta en concreto al baloncesto y al descubrimiento del talento deportivo, hay un curioso e interesante estudio ${ }^{11}$ donde se relaciona el éxito en este deporte y la habilidad deportiva en aquellos individuos que poseen un índice más bajo entre la longitud del dedo índice y el anular (I 2D/4D). Parece ser que este índice se aproxima a una valoración de la tasa de andrógenos durante la vida fetal, y eso se correlaciona con la velocidad de carrera y con una percepción espacial determinada. Es un método sencillo y fácil de evaluar. Lo que no sabemos - porque nadie se ha dedicado a valorarlo- es si la evidencia científica posee la suficiente fuerza como para que el presentado índice sea determinante en la selección del talento deportivo o sea una mera anécdota. Con la corta experiencia de las mediciones en fútbol y baloncesto base y profesional en el FC Barcelona, debo decir que si bien este índice es positivo en jugadores que son verdaderos talentos, hay algunos otros que sí están compitiendo a elevado nivel pero no son comparables, y por otro lado existen sujetos con el índice señalador de talento pero que no parece que vayan a salir. ¿Son estos últimos aquellos que no se ha sabido potenciar en su máxima expresión? Siempre quedará la duda. El tiempo y el seguimiento de diversos equipos nos darán la solución a esta hipótesis.

\section{Condiciones PSICOSOCIALES}

Como indica muy bien Lorenzo ${ }^{12}$ respecto a los factores que condicionan la detección, el desarrollo y la selección del talento en el fútbol, se ha sugerido que los jugadores exitosos tienen características psicológicas determinadas que favorecen el que alcancen los resultados deseados, señalando entre otras características la habilidad para superar la ansiedad y los obstácu- 


\section{REVISIONES}

Tabla III Diferentes particularidades según la filosofía de dos escuelas de baloncesto base en las que predomina el interés de buscar la victoria como objetivo principal o la de formar al jugador de una forma integral con objetivos a largo plazo; modificada levemente de la idea de Horst Wein para el fútbol ${ }^{10}$

\begin{tabular}{|c|c|}
\hline Objetivo: GANAR & Objetivo: FORMAR \\
\hline Importancia a jugadores con elevadas capacidades físicas & $\begin{array}{l}\text { Preferencia por jugadores con perspectiva en la capacidad de jugar la } \\
\text { pelota }\end{array}$ \\
\hline Trabajo de la táctica desde benjamines en los partidos & En los partidos se evalúa el grado de uso de la técnica \\
\hline Se busca la profundidad en juego de ataque & No hay prisa en el juego. Se pretende mantener la posesión del balón \\
\hline Juego rápido, pases largos & $\begin{array}{l}\text { Todos los jugadores mueven el balón. El portero sirve con la mano. } \\
\text { Pases cortos }\end{array}$ \\
\hline Pocos cambios de orientación en las acciones ofensivas & $\begin{array}{l}\text { Se cambia con frecuencia la orientación del juego con el objeto de } \\
\text { crear espacios }\end{array}$ \\
\hline $\begin{array}{l}\text { Pocas intenciones de construir juego. La pelota pasa directo del base } \\
\text { a los jugadores, que pueden anotar rápidamente o con gran seguridad }\end{array}$ & $\begin{array}{l}\text { La pelota circula desde la dirección de juego por los jugadores que } \\
\text { buscan posiciones diversas. Juego basado en la comunicación y en la } \\
\text { cooperación }\end{array}$ \\
\hline $\begin{array}{l}\text { Especialización prematura de las demarcaciones. Juegan los mismos } \\
\text { y poco los suplentes }\end{array}$ & $\begin{array}{l}\text { Todos tienen oportunidad de experimentar varios puestos. Juegan } \\
\text { todos, con independencia de la calidad }\end{array}$ \\
\hline El jugador obedece al técnico que manda e instruye desde la banda & $\begin{array}{l}\text { El jugador realiza la próxima acción en función de su propia capacidad } \\
\text { de percepción y toma de decisión, sin intervención del formador } \\
\text { que estimula y no instruye }\end{array}$ \\
\hline Se juega prematuro basket de adulto, aros, campo, reglamento... & $\begin{array}{l}\text { En cada fase de evaluación del niño se adapta el juego a las } \\
\text { capacidades físicas y mentales }\end{array}$ \\
\hline $\begin{array}{l}\text { Para poder ganar prevalece en los entrenamientos la aplicación de la } \\
\text { metodología tradicional }\end{array}$ & $\begin{array}{l}\text { Para formar mejor al jugador debe prevalecer en las prácticas la } \\
\text { aplicación de la metodología activa }\end{array}$ \\
\hline
\end{tabular}

los que vayan surgiendo, la confianza en uno mismo, la competitividad, la motivación intrínseca, la habilidad para evitar distracciones o la capacidad para establecer objetivos y alcanzarlos. Existe un acuerdo generalizado sobre el hecho de que alcanzar dichos resultados está condicionado a un elevado compromiso, determinación y perseverancia para superar las dificultades. Aspectos basados en unas características observadas en estos sujetos ${ }^{13}$ :

- Disciplina, entendida ésta como la capacidad del joven deportista de cumplir con las exigencias que le imponía la actividad deportiva, así como la voluntad para aceptar ese sacrificio, especialmente en su vida personal.

- Compromiso, representando los aspectos motivacionales que llevaban a los jóvenes deportistas a comprometerse con su carrera como jugadores de fútbol.

- Resistencia, entendida como la capacidad de continuar o superar las adversidades, tanto de tipo personal como contextual.

- Apoyo social, entendido éste tanto desde el punto de vista emocional, informacional como económico.

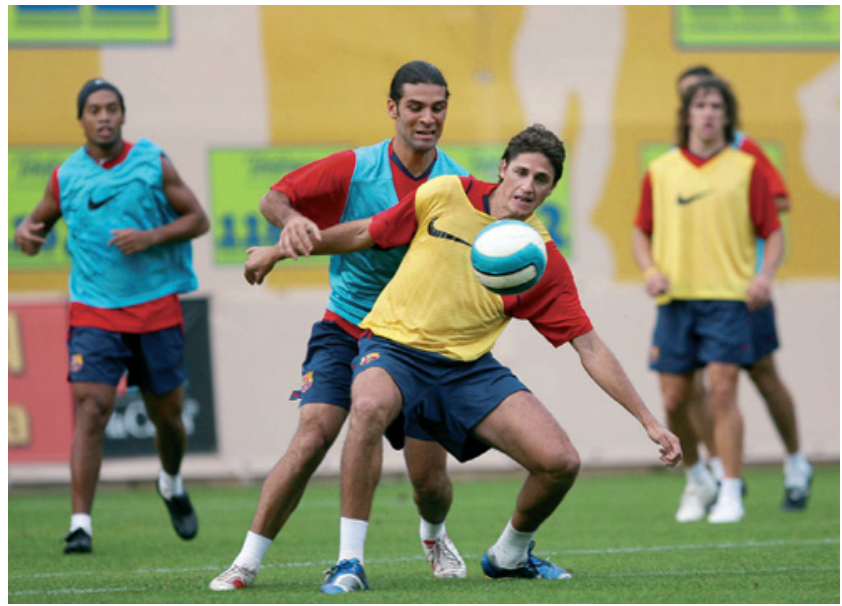

El deportista experto evalúa cada aportación de ambos lados de la balanza que mide el grado de compromiso, los beneficios obtenidos y la inversión expuesta. La diversión, la inversión personal de tiempo, ilusiones, renuncias, el alcanzar un mayor dominio del deporte, el sentirse más competente, ser reconocido social y económicamente y, por último -pero 
no menos importante-, la aportación de las alternativas contextuales y coacciones sociales como puede ser la influencia de la familia. En esto se basa la teoría del compromiso deportivo $^{14}$, que se estructura a partir de los 8-9 años de trabajo. Es decir, sobre los 18, que es cuando el individuo puede evaluar si merece la pena invertir en el camino del deporte o no. En este período, pero sobre todo en los años de inicio, es de gran importancia el grupo de amigos del jugador. Cuando se hace la historia deportiva de un jugador, la gran mayoría de las veces éste practica deporte porque "sus amigos lo practicaban”. Esta interacción con los amigos permite a los jugadores expertos dedicarse a su deporte durante el tiempo libre, lo que, por otra parte, conlleva un aumento en el tiempo de experimentar recursos y situaciones. Sería el ejemplo del baloncesto o el fútbol de la calle que, sobre todo, se practican en Estados Unidos y Suramérica, respectivamente.

\section{EDAD BIOLÓGICA Y CRONOLÓGICA}

En un mismo campo de juego puede haber jugadores con una diferencia de edad de hasta 2 años o algo más. Las categorías infantil y cadete son las que ven repercutidos con más énfasis sus resultados por este aspecto. Es importante reconocer a los individuos con una edad de maduración superior o inferior en un equipo para que puedan evolucionar bien. No se debe olvidar que la maduración ósea no es la maduración mental, y no es bueno que jueguen en categorías en las que las edades no les competen, al menos en general, pues excepciones siempre existen. Recordemos que las inquietudes, los problemas, las materias de las que hablar en esas edades son muy diferentes de los 12 a los 16 años. Un buen planteamiento es que, al menos un día a la semana, el jugador "desfasado" con respecto al grupo entrene con la categoría que le corresponde. Es una manera de mantener unas referencias adecuadas entre el individuo y el grupo. Recordemos que a partir de los 16 años las cualidades físicas debidas al proceso de maduración ya no tendrán fuerza y sólo será determinante, en el aspecto físico, el entrenamiento realizado. Si por un exceso de cualidades en ese sentido no se trabajaron en su máxima expresión, o se calibraron mal por hallarse por debajo, alcanzarán la categoría de juveniles con un déficit que puede ser determinante y difícil de corregir en un corto período de tiempo, pudiéndose perder un sujeto en el que se ha invertido ilusión, tiempo y -por qué no- un dinero. La edad biológica puede confundirnos y demostrarnos un talento cuando en realidad no lo es tanto y viceversa. Por eso es necesario establecer la edad biológica del deportista y saber en qué momento evolutivo se halla. En cual-

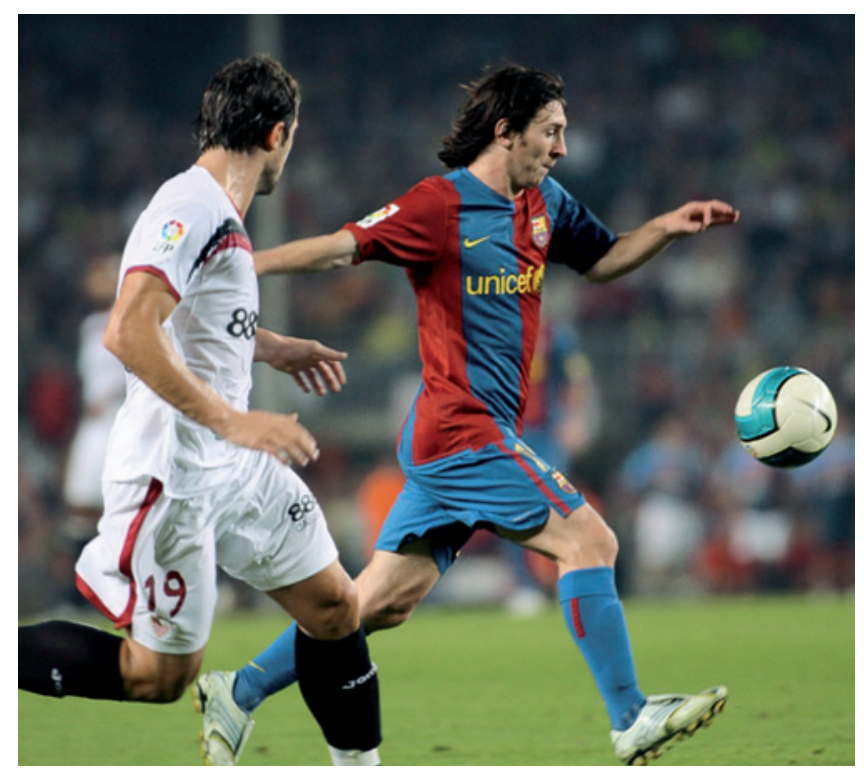

quier caso -y es una opinión consensuada-, en un jugador con las limitaciones de la ausencia de experiencia por su juventud, las habilidades técnico-tácticas, la facilidad para tomar decisiones positivas, la habilidad social con los compañeros dentro del terreno, son condiciones más importantes que las que devienen de condicionantes físicos. En el momento actual, la mejora de las cualidades físicas puede alcanzarse de una forma progresiva y bien estructurada hasta un nivel de rendimiento físico del más alto nivel. Es cierto que el camino será más arduo para aquel deportista quizás menos agraciado genéticamente, pero no debe asustarnos la elección, pues duro lo es para todos.

Existe una relación muy significativa entre la fecha de nacimiento y el éxito deportivo observada desde hace un tiempo, determinada por la iniciación de la temporada deportiva o del período escolar, por la cultura deportiva del país, etc. Por esa razón, la fecha de nacimiento se ha considerado como uno de los diversos factores en la selección de los talentos deportivos para diversos deportes. Un aspecto interesante, relacionado con la fecha de nacimiento y el rendimiento deportivo, es el de la edad relativa (RAE, relative age effect). El RAE determina la diferencia de edad entre individuos de un mismo grupo de edad. Se ha identificado como un factor que puede tener cierta influencia sobre aspectos muy diversos de carácter sociológico y psicológico o relacionado con el rendimiento deportivo y la actividad física. Así, según diversos autores, este efecto parece influir de manera muy decisiva en la selección del talento deportivo y en su proyección hacia un nivel profesional. Una supuesta selección del deportista por nacer en una determinada época del año quizá subestimaría a futuros talentos cuyo estado de madu- 


\section{Figura 3 \\ Diferencias en el porcentaje de nacimientos por trimestres entre el baloncesto base de dos equipos, $y$ el baloncesto profesional español y el de la NBA (ambas ligas sin extranjeros). Obsérvese que en los clubes hay una tendencia a premiar los dos primeros trimestres sobre los segundos, mientras que en el ámbito de ACB-LEBI y LEB2 persiste una tendencia y no ocurre en la NBA.}

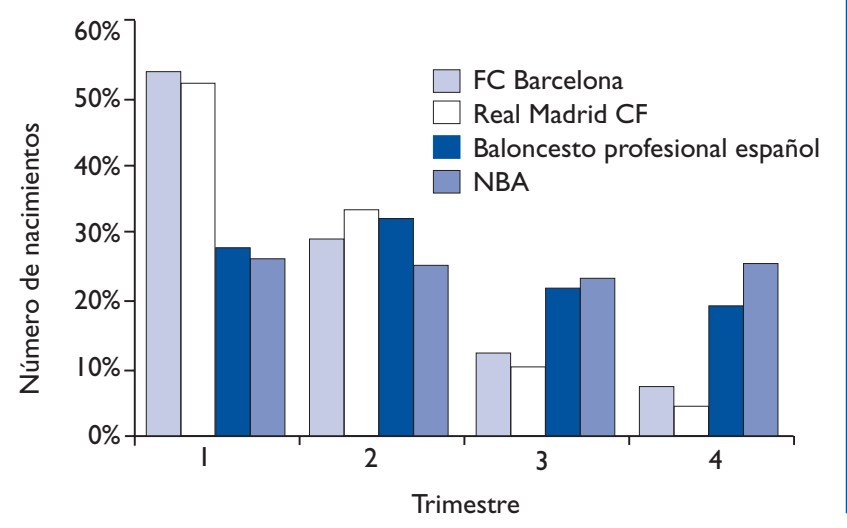

ración y desarrollo son inicialmente inferiores. En la figura 3 se presenta el efecto de la edad en la selección en baloncesto en dos equipos de nuestro país frente al global de la liga profesional (ACB-LEB 1 y 2) y la NBA. Se observa un efecto relativo de la edad en los jugadores de baloncesto de nivel profesional español y de mayor intensidad en los jugadores pertenecientes al deporte base. No existe este efecto relativo de la edad en el baloncesto profesional americano. Por otro lado ${ }^{12}$, y respecto a este tema, el nivel de rendimiento de un individuo es el resultado de sus capacidades y de su motivación. El grado de su motivación tiene un impacto importante sobre la calidad de su aprendizaje y su rendimiento. Un jugador nacido al principio del año obtendrá, como media, mejor rendimiento que otro jugador nacido al final del año, además de jugar menos y ser menos veces convoca$\mathrm{do}^{15}$. Por eso, esta situación deben evaluarla los técnicos, los científicos y los políticos del deporte para establecer las herramientas y los sistemas adecuados de selección, de seguimiento y de entrenamiento de los deportistas con el objeto de optimizar los jugadores que podamos disponer en el futuro.

Por suerte, en los deportes de equipo se goza de diversas características que determinan el rendimiento óptimo, donde las cualidades físicas poseen un valor importante pero que no deja de ser un complemento de las cualidades técnicas, tácticas, psicológicas o sociológicas. Por otra parte, es el carácter y la profesionalidad del deportista lo que ayudará a alcanzar los objetivos cuando existan ciertas deficiencias físicas.
Tabla IV Algunas consideraciones para un entrenador de talentos

Las cualidades técnicas son más importantes que las físicas en la selección del talento

Las deficiencias de condición física se alcanzan con un trabajo bien programado

Establecer objetivos elevados para estimular el aprendizaje

Introducir la excelencia en los entrenos. Nunca un entreno mediocre

Enseñar a aprender de los errores, de los éxitos y del fracaso

Facilitar la incorporación de aspectos de conocimiento de la salud y el rendimiento deportivo. Iniciar en la profesionalidad

Aprender a trabajar en condiciones especiales, para alcanzar deportistas adaptables

No poner por delante la mejora de una técnica por encima de la adquisición de técnica

Introducir elementos de juego de otros deportes para mejorar habilidades determinadas

Los grandes deportistas ofrecen más de lo que el entrenador solicita, y los grandes entrenadores siempre hacen más de lo que el deportista espera

El buen profesional, agraciado por una buena condición física, debe vigilar su dieta, el descanso, el trabajo de condición física adaptado a su perfeccionamiento técnico y táctico. Cada día más el jugador debe preocuparse de su estado de salud. Desde las categorías inferiores. La buena alimentación y el descanso adecuado facilitarán que el estrés físico del entreno se transforme en la carga de trabajo adecuada para un buen desarrollo corporal y como individuo. La calidad de la inversión en la infancia y en la adolescencia es lo que hallaremos en el período de mayor rendimiento profesional.

La imposición de valores y talentos debe ser a largo plazo. Se pueden esperar grandes rendimientos en categorías inferiores, pero no debe ser una exigencia. Si éstos llegan, mucho mejor, pero debe enseñarse el camino de la excelencia en todos los ámbitos del deporte elegido, y, por qué no, de la vida. En ese objetivo nos hallamos todos, los entrenadores, los servicios médicos, los preparadores físicos, las directivas de los clubes, los padres y los compañeros.

En este artículo no se ha pretendido dar soluciones ni normas para encontrar el jugador de talento, sino ofrecer algunas ideas que consideramos es obligado tener en cuenta. Cada club debe hacer su propia selección en función de sus objetivos y necesidades. En la tabla IV se indican algunas ideas para los entrenadores que trabajan con futuros deportistas de gran nivel. 
1. Hahn E. Entrenamiento con niños. Barcelona: Martínez Roca; 1988.

2. Cohen Grinvald R. Fútbol: detección y desarrollo del talento deportivo. Disponible en: http://www.efdeportes.com/efd10/talent10.htm

3. Simon HA, Chase WG. Skill in chess. American Scientist. 1973;61:394-403.

4. Newell A, Rosembloom PS. Mechanisms of skill acquisition and the law of practice. En: Anderson J, editor. Cognitive skills and their acquisition. Hillsdale, NJ: Erlbaum; 1981. p. 1-55.

5. Ericsson KA, Krampe RT, Tesch Römer C. The role of deliberate practice in the acquisition of expert performance. Psychological review. 1993;100:363-406.

6. Horton S. Facilitating expertise. The role of expert coach. Tesis doctoral no publicada. Canada: Queen's University; 2003. En: Jiménez S, Lorenzo Calvo J, Lorenzo Calvo A, editores. Factores asociados al desarrollo de la pericia en los entrenadores. Disponible en: http://www.efdeportes.com/efd88/pericia.htm

7. Baker J, Horton S, Robertson-Wilson J, Wall M. Nurturing sport exercise: factors influencing the development of elite athlete. Journal of Sports Science Medicine. 2003;2;1-9.

8. Bompa TO. Theory and methodology of training: The key to athletic performance. Dubuque, Iowa: Kendall/Hunt Publishing Company; 1983. p. 35-41, 99-203, 207-12.
9. Côté J, Hay J. Children's involvement in sport: A developmental perspective. En: Silva J, Stevens D, editors. Psychological foundations of sport. Boston, MA: Merrill; 2002. p. 484502 .

10. Wein Horst. Developing youth soccer player. Stanningley, Leeds (UK): Human Kinetics. 2000.

11. Manning JT, Bundred PE, Taylor R. The ratio 2nd and 4th digit length: a prenatal correlate of ability in sport. En: Reilly T, Marfell-Jones M, editors. Kinanthropometry VIII. London: Roudletge; 2003. p. 165-74.

12. Lorenzo Calvo A. Detección, desarrollo y selección del talento en fútbol, factores que condicionan el desarrollo del futbolista. Guía didáctica del Master Universitario de preparación física en fútbol. Madrid: Real Federación Española de Fútbol; 2005.

13. Holt NL, Dunn JG. Toward a Grounded Theory of the psychosocial competencies and environmental conditions associated with soccer success. Journal of Applied Sport Psychology. 2004;16:199-219.

14. Carpenter PJ, Scanlan TK, Simons JP, Lobel M. A test of the Sport Commitment Model using structural equation modeling. Journal of Sport \& Exercise Psychology. 1993;15:119-33.

15. Vaeyens R, Philippaerts RM, Malina RM. The relative age effect in soccer: A match-related perspective. Journal of Sports Sciences. 2005;23:747-56.

\section{Bibliografía general}

Côté J. The influence of the family in the development of talent in sports. The Sport Psychologist. 1999;13:395-417.

Esteva S, Drobnic F, Puigdellivol J, Serratosa L, Chamorro M. Fecha de nacimiento y éxito en el baloncesto profesional. Apunts. Medicina de l'esport. 2006;41,149:25-30.

Lorenzo Calvo A. ¿Detección o desarrollo del talento? Factores que motivan una nueva orientación del proceso de detección de talentos. Apunts d'Educació Física. 2004;71:23-8.

Lorenzo Calvo A. La detección del talento en los deportes colectivos. Kronos: revista universitaria de la actividad física y el deporte. 2002;1:15-23.

Manonelles P. Edad cronológica como factor de elección de jugadores de las selecciones españolas de baloncesto de formación. Archivos de Medicina del Deporte. 2003;96:321-8.
Matsudo VKR. Prediction of future athletic excellence. En: Bar-Or O, editor. The Child and Adolescent Athlete. Oxford: Blackwell Science; 1996. p. 92-109.

Pancorbo A. Detección de talentos deportivos. Medicina del deporte y ciencias aplicadas al alto rendimiento y la salud. Caxias do Sul, Brasil: EDUCS Editoria da Universidade de Caxias do Sul; 2002. p. 258-91.

Reilly T, Gilbourne D. Science and football: a review of applied research in the football codes. Journal of Sport Sciences. 2003; 21:693-705.

Ruiz LM, Sánchez F. Rendimiento deportivo: claves para el aprendizaje. Madrid: Gymnos; 1997. 